\title{
A System Architecture of Service Fusion Engine for Information of Physical World
}

\author{
Ting Wang, Hualiang Zhang, Peng Zeng \\ Department of Industrial Control Network and System \\ Shenyang Institute of Automation Chinese Academy of Sciences \\ Shenyang, Liaoning, 110016, China \\ E-mail: wang.ting@microcyber.cn
}

\begin{abstract}
Due to the physical space and information space have the complex coupling, building the information association of dynamic service become the key issue of the future Internet developing. The existing Internet theory and method is difficult to meet the challenges. This paper explained the architecture of the service fusion engine for the physical world information. It built a set of the ontology based real environment, and overcome uncertainty, high hybrid and interrelated of the physical information. It uses the physical information characteristic for information search and predicts of user behavior, realized user demand service composition. Finally, the service fusion engine formed a set of ontology knowledge based physical information and service inference rules form the environment knowledge.
\end{abstract}

Keywords-information of physical world; service fusion; inference ;

\section{INTRODUCTION}

With the rapid development of Internet, in the future The future of the world's information process will be human society to the physical world expansion, The Information Of Physical world(IOP) will become the main source of information, The amount of information will be far exceeds the available information in human society[1].

IOP has massive heterogeneous, temporal and spatial multi-dimensional association, and other unique characteristics of dynamic real time. With the deepening of human participation, IOP perception will develop from the traditional human-computer dual-element to three-element of human-computer-thing fusion model. Three-element of human-computer-thing fusion model will Continuous generation and accumulation of huge amount of data. On the one hand, these data have a typical massive, heterogeneous, time and space, data flow and sequence characteristics, on the other hand, physical space and the information space of the strong correlation, uncertainty and highly mixture bring a new set of challenges about the build of IOP association and service dynamic generation, the existing Internet theory and method is difficult to meet the challenges, many basic problems to be solved urgently at the same time.

We have many challenges:

(1) Massive IOP heterogeneous leads to inefficient physical device interoperability.

(2) Data format conversion cost a great quantity resource because all kinds of the terminal communication protocol [2]

(3) Real time physics information has high search difficulty, searching efficiency is very low [7].
(4) User oriented application demand service push the lack of intelligence, the lack of individuality [8].

So we urgently need a thorough analysis of the physical space and cyberspace coupling mechanism. We need an in depth analysis of physical information underlying commonality and knowledge organization, and we need to predict the future environmental changes to provide efficient personalized service for the social life. This architecture will build a perceptual interaction, autonomous and efficient service platform, according to IOP uncertainty, high hybrid and interrelated. Due to the existing information service based on Web mechanisms is difficult to adapt to the real environment for the user to provide spatiotemporal correlation adaptive service challenge, the architecture of the main study of IOP in the real environment spatiotemporal correlation service synthesis technology. We descripted the IOP follow standardization standard, analyzed of the uncertainty, high hybrid and interrelated, and then establish a set of physical information service fusion engine based ontology knowledge and situational inference, eventually formed a cross industry across the field, perceived interaction, autonomous and efficient service platform.

\section{RELATED WORKS}

The recent related research projects focus on the smart space, semantic web applications. These systems used the context-aware module to control sensor and actuator based on the information of user's situation, environment [3]. [4] Proposed an architecture named the Context Broker based semantic web and ontology. The paper's author worked in the Apple and developing the famous SIRI sound assistant. [5] Proposed an intelligent system with some sensors in each domain to aware the environment information for building energy saving and context-aware smart services. Due to the system used sensors to monitor user's behavior, the result of energy saving is dependent on the number of sensors. Userdevice interaction based on context-aware: A situation aware technology is aware of the user's state and environment and modifies system behavior based on the information [6].

\section{ARCHITECTURE}

In this paper, the project focus to construct a perceptual interaction, autonomous and efficient service platform as the goal, according to IOP information uncertainty, high hybrid and interrelated analysis and knowledge building as a starting point, focus on solving the following problems: the physical space and information space complex coupling 
leads to physical information associated with the service dynamically generated difficulties. The system is composed of four parts: a IOP information modeling and unified characterization and low overhead release part, the real-time information of IOP vertical search engine part, and a IOP oriented information ontology and ontology based reasoning mechanism of Library Service part. Figure 1 shows the Service Fusion Engine architecture.

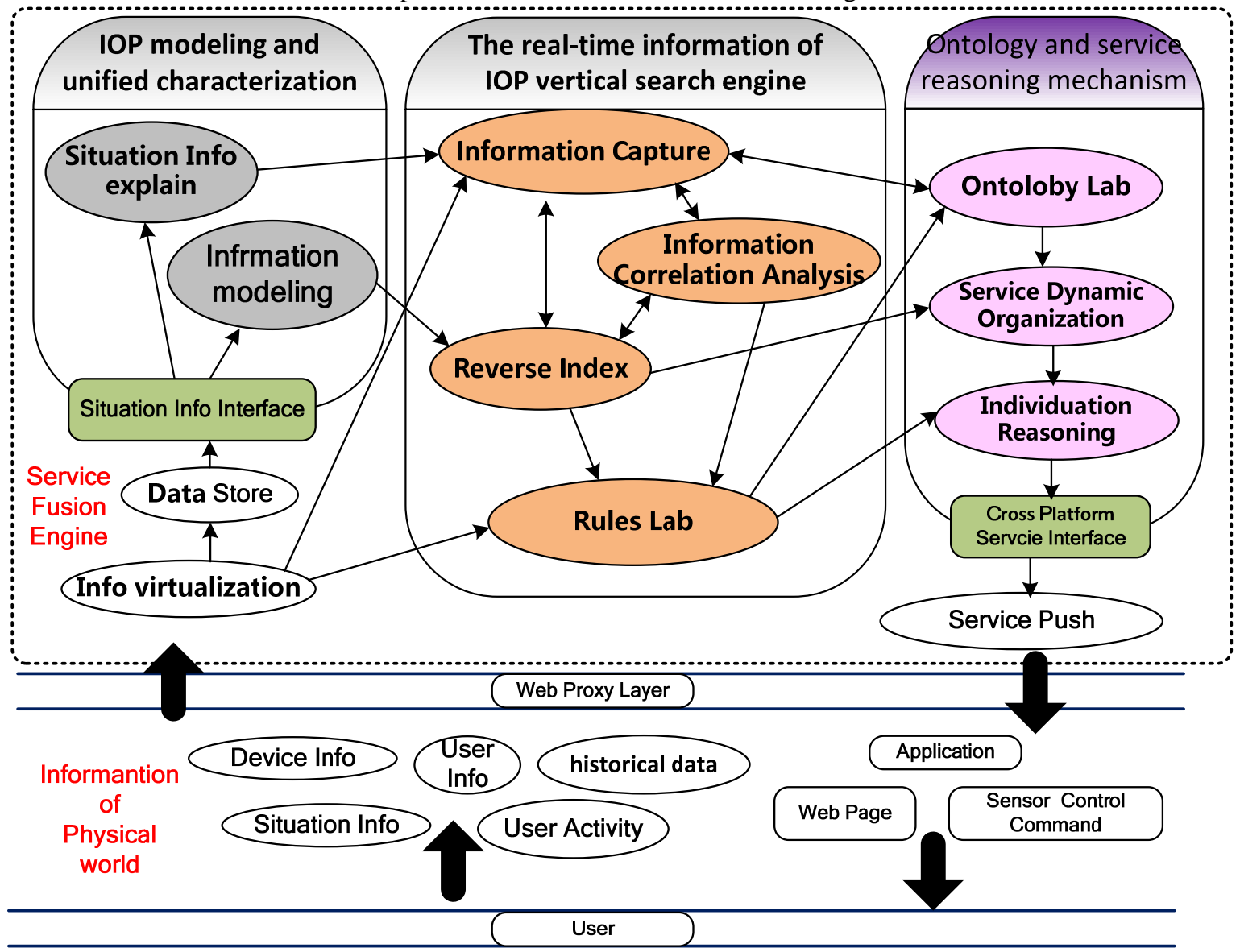

Figure 1 Service Fusion Engine architecture

A. IOP modeling and unified characterization and low overhead release

The part analyzes the IOP node information diversification characteristics and researches IOP key factors and temporal and spatial characteristics of information mapping technology. The part is composed of three functions.

1) According to the IOP heterogeneous, loosely coupled, dynamic characteristics of the unified description of the information in the extensibility and reuse;

2) IOP modeling and unified representation and automatic conversion;

3) In view of the physical release of information with low cost and low power requirements, process low hardware overhead of Web Services embedded device;

\section{B. The real-time information of IOP vertical search engine}

This part includes the module of a new oriented physical gateway and interoperability adapter crawler to crawl the embedded dynamic information, and the module of fusion at physical information and Internet information in large scale distributed caching system. The part supported streaming data, text data and kinds of the real-time data's development plugin. It Implemented read/write requirement of ontology library construction and service data request support reasoning mechanism.

1) Analyzed IOP features of the transform process and capture IOP real-time dynamic information;

2) Aiming at the sensor networks heterogeneous and addressing technology analysis and research of the depth and breadth traversal of IOP grasping algorithm;

3) According to the IOP capture results of the data analysis, structure optimization, key information extraction and classification of IOP transformation;

4) According to the IOP conversion of information results, over statistic, weight, inverting built the multilevel distributed index. Figure 2 shows the IOP vertical search engine principle. 


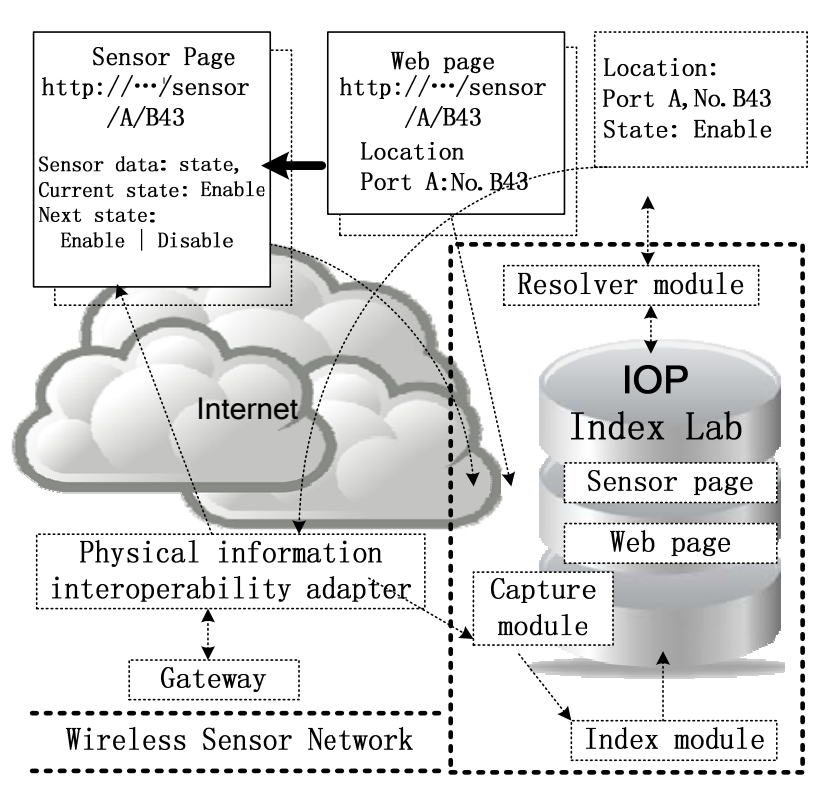

Figure 2. IOP vertical search engine principle

\section{IOP ontology and service reasoning mechanism based} ontology lab

This part included a set of module, it set the ontology base entities, attributes, and the concept of justice association rules, abstract the conventionalized conceptual category, build the distributed ontology database storage model, then design the ontology mapping, matching, aligned with the merging algorithm. The algorithm implements the ontology library distributed storage system.

Aforementioned module use ontology base entities, attributes, the axiom and the example association to establish the rule lab, and build the atomic-rule based the deterministic application scene's dynamic rule matching algorithm to form the forward-backward inference mechanism. On the ontology entity, the reason module predicts user behavior based dynamic probability model, and using the model to establish the optimal Bayesian network. Then reason module design the uncertainty reasoning and implicit rule reasoning algorithm, promote rules to generate a robust inference engine.

1) According to the key elements of IOP information, analyzed ontology concept, functions, axioms, and the association;

2) According to the IOP ontology relation results from a combination of the user service requirements design services the forward-backward inference process;

3) According to user service forward backward reasoning process and the practical application the uncertainty in the scene to predict user behavior, and design the dynamic probability mode;

4) Based on the dynamic probability model to design the uncertainty of fuzzy reasoning algorithm of scene;

5) Evaluate the accuracy of based scene in ontology construction and the result of fuzzy reasoning.

\section{IMPLEMENTATION}

We have implemented some parts of our architecture. We have implemented Greenplum database to store the structuring data, and the Hbase to store the NoSQL data, Map-Reduce and the Storm co-worked to process the blended data. We collected the sensor data, labeled them with the semantic tab follow the SIA-MAP, which is the ontology lab and built by us. The sensor information converted virtual information of the Virtual Sensor and the Sensor Group after the labeling. The Web Server published the information of the Virtual Sensor and the Applications subscribe the information which it needed. The Compute platform scheduled the Pub-Sub Jobs to match the application needs. We implemented the smart domestic equipment monitor based the platform. Figure 3 shows the technology view of our system.

\section{CONCLUSIONS AND FUTURE WORKS}

This paper proposed IOP modeling and unified characterization technique, and built a set of IOP information ontology based semi-automatic / automatic construction technology. At the same time, we designed service reasoning algorithm for physical space user, and built basic theory system about the physical information interoperability and service composition engine. The architecture implemented a set of key technology to support IOP integration and open information service interoperability, information, service reasoning. And there is some work being done and will be done. We expect that our work will contribute to the development of building an open service infrastructure and an open sensor cloud Ecosystem for.

\section{ACKNOWLEDGMENT}

The authors acknowledge the financial support of the Strategic Priority Research Program of the Chinese Academy of Sciences under Grant No.XDA06020602.

\section{REFERENCES}

[1] L. Atzori, A. Iera, and G. Morabito, "The Internet of Things: A survey,” Comput. Netw., vol. 54, 2010, pp.2787-2805.

[2] M. Yuriyama and T. Kushida."Sensor-Cloud Infrastructure”.The 13th International Conference on Network-Based Information Systems, 2010.

[3] Chu Weijie; Mo Tong; Cui Jie; Wang Yuan; Xu Jingmin; Li Weiping; Lin Huiping, "A Context-Aware Services Development Model”, Service Sciences (IJCSS), 2012 ,pp 194-199.

[4] Harry Chen, Tim Finin, Anupam Joshi. "Semantic Web in the Context Broker Architecture" Pervasive Computing and Communications,2004, pp.277-286.

[5] J. Byun, I. Hong, B. Kang, and Sehyun Park. "A Smart Energy Distribution and Management System for Renewable Energy Distribution and Context-aware Services based on User Patterns and Load Forecasting" Consumer Electronics, IEEE Transactions on, Vol.57, 2011, pp.436-444.

[6] A. Krause, A Smailagic and D. P. Siewiorek, "Context-Aware Mobile Computing: Learning Context-Dependent Personal Preferences from a Wearable Sensor Array," Mobile Computing, IEEE Transactions on, vol.5, 2006, pp.113-127

[7] Bröring, A., J. Echterhoff, S. Jirka, I. Simonis, Everding, C. Stasch, S Liang, \& R. Lemmens (2011): New Generation Sensor Web Enablement. Sensors, 11(3), pp. 2652-2699. 
[8] P. D. Reyes, J. Favela and J. C. Castillo, ”Uncertainty Management in Context-Aware Applications: Increasing Usability and User Trust”,Springer Wireless Personal Communications, vol. 56, no. 1,2011, pp. 37-53.

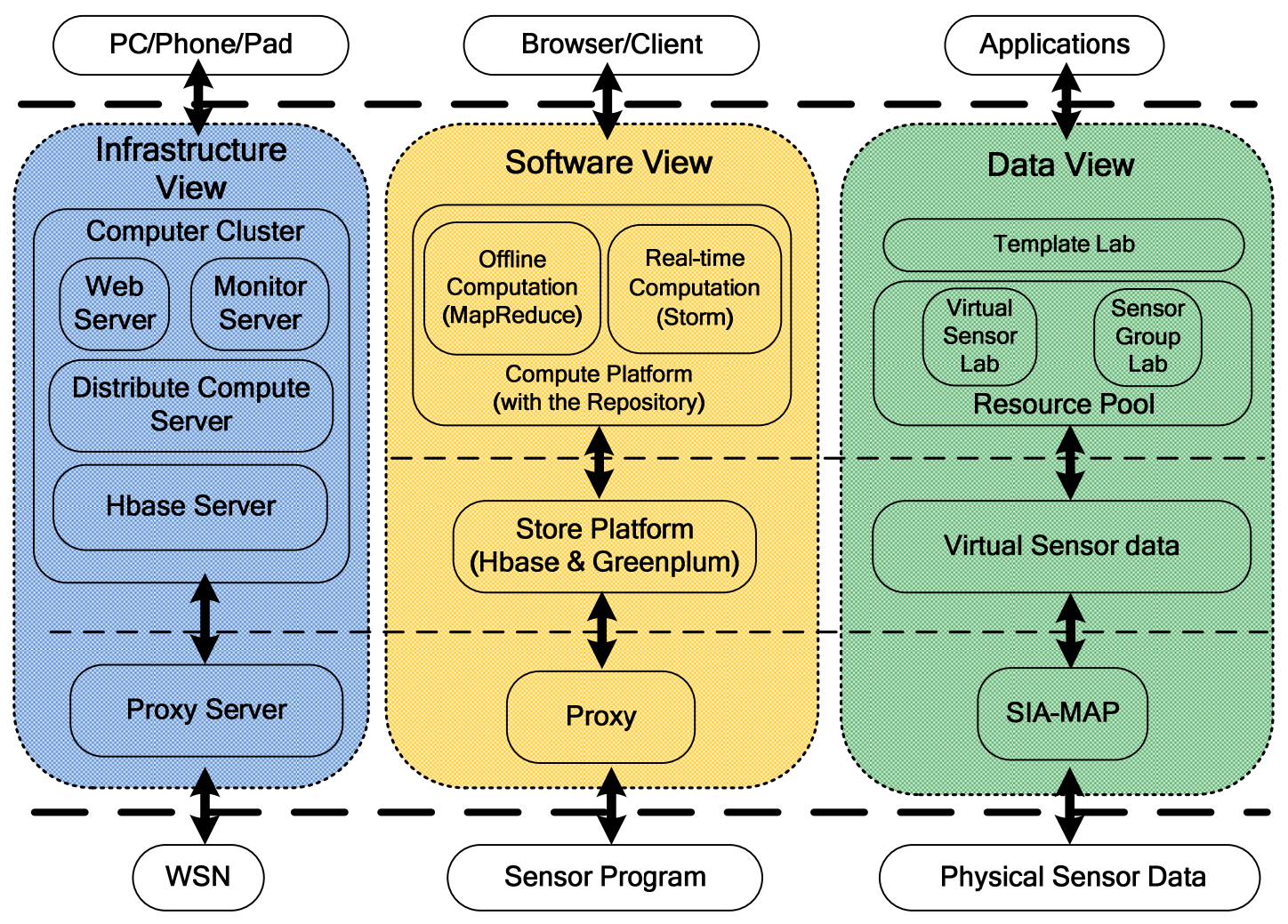

Figure 3. Service Fusion Engine Technology View 